\title{
Resultados e interpretación al aplicar la técnica de matriz de riesgo en braquiterapia
}

\author{
Results and interpretation for the application of the risk \\ matrix method in brachytherapy
}

\author{
Diana Carolina Herrera ${ }^{1}$, Mauricio Arciniegas Álvarez², José Alberto Gómez ${ }^{3}$ \\ Citación: D. C. Herrera, M. Arciniegas y J. A. Gómez, "Resultados e interpretación al aplicar la \\ técnica de matriz de riesgo en braquiterapia", Revista Investigaciones y Aplicaciones Nucleares, $\mathrm{n}^{\circ}{ }^{3}$, \\ pp. 5-11, 2019. https://doi.org/10.32685/2590-7468/invapnuclear.3.2019.506
}

Recibido: 30 de septiembre de 2019 Aceptado: 23 de diciembre de 2019 Publicado en línea: 30 de diciembre de 2019

Doi: https://doi.org/10.32685/25907468/invapnuclear.3.2019.506

Palabras clave: evaluación del riesgo, braquiterapia, seguridad radiológica.

\section{RESUMEN}

El objetivo de este trabajo es presentar los resultados obtenidos al aplicar la técnica de matriz de riesgo, con la aplicación SEVRRA, en braquiterapia, y además las precauciones que se deben tomar en su aplicación y en la interpretación de los resultados. Para este fin se realizó el análisis de riesgo de una instalación de braquiterapia de alta tasa de dosis utilizando el software SEVRRA y con una herramienta desarrollada en base en el método de matriz de riesgo en una hoja de cálculo. Los resultados del segundo cribado mostraron discrepancias entre el perfil de riesgo obtenido con la aplicación SEVRRA y la herramienta propia. El porcentaje de sucesos iniciadores con riesgo alto con la aplicación SEVRRA fue de 6\%, y con la herramienta propia de $14 \%$. Luego de un análisis de cada suceso se observó que las discrepancias se deben a que la ponderación de la robustez de las barreras de seguridad en SEVRRA es inconsistente con las definidas en la técnica de matriz de riesgo. Estos resultados tienen profundas implicaciones en función de la aceptabilidad del riesgo planteada en la metodología. Adicionalmente, se encontró que la metodología de matriz de riesgo no tiene en cuenta los riesgos inherentes a la práctica. A pesar de las inconsistencias observadas, se reconoce el potencial de la herramienta SEVRRA para la evaluación de riesgo de instalaciones radiactivas, por ser de fácil uso; sin embargo, se recomienda la revisión de la robustez de algunas barreras, con el fin de obtener el perfil de riesgo de acuerdo con la metodología. De esta forma en base a los resultados, se podrán priorizar las medidas que deben adoptarse para garantizar la seguridad radiológica de las instalaciones.

\section{ABSTRACT}

The objective of this study is to present the results obtained with the risk matrix method using the SEVRRA application in brachytherapy and the precautions that should be taken when applying this approach and interpreting the results. Risk analysis was performed for a high-dose-rate brachytherapy facility using the SEVRRA software and a tool developed based on the risk matrix method in a spreadsheet. The results of the second screening showed discrepancies between the risk profiles obtained with the SEVRRA application and with the custom tool. The percentages of high-risk initiating events obtained with the

\footnotetext{
${ }^{1}$ Universidad Nacional Abierta y a Distancia

${ }^{2}$ Hospital Universitario San Ignacio

${ }^{3}$ Radsolutions SAS

Email de correspondencia: diana.herrera@unad.edu.co
} 
Keywords: risk assessment, brachytherapy, radiological safety
SEVRRA application and the custom tool were $6 \%$ and $14 \%$, respectively. After analysis of each event, it was observed that discrepancies occur because the weighting of the robustness of the safety barriers in SEVRRA is inconsistent with that defined in the risk matrix method. These results have profound implications regarding the risk acceptability set in the method. Additionally, it was found that the risk matrix method does not consider the risks inherent to practice. Despite the observed inconsistencies, the potential of the SEVRRA application for risk assessment of radiotherapy facilities is recognized, given that this application is easy to use; however, review of the robustness of certain barriers to obtain the risk profile according to the risk matrix method is recommended. Based on the results, it will be possible to prioritize measures that must be adopted to guarantee the radiological safety of radiotherapy facilities.

\section{INTRODUCCIÓN}

$\mathbf{L}$ a braquiterapia de alta tasa de dosis (braquiterapia HDR) se realiza con radiaciones ionizantes, de modo que el tejido del paciente es irradiado directamente con tasas de dosis $>12 \mathrm{~Gy} / \mathrm{h}$ [1]. La aplicación de esta técnica implica una serie de etapas en las que participan distintos profesionales, lo que incrementa el riesgo de tener un incidente o accidente. Por esta razón, las normas básicas internacionales de seguridad para la protección contra la radiación ionizante y para la seguridad de las fuentes de radiación (BSS) [2] disponen que en este tipo de prácticas se realice un análisis de riesgo, consistente en investigar los riesgos de los posibles eventos que impliquen potenciales exposiciones, y en concordancia con los resultados, se adopten medidas para prevenirlos.

Teniendo en cuenta este requerimiento, en Colombia, a partir de la vigencia de la Resolución 90874 del 2014 del Ministerio de Minas y Energía [3], es requisito realizar el análisis de riesgo en instalaciones de braquiterapia HDR, con el fin de obtener la autorización para el manejo de fuentes radiactivas. Desde que entró en vigencia esta reglamentación, el Organismo Internacional de Energía Atómica ha impartido capacitaciones al personal del ente regulador y al de las instalaciones radiactivas. En las capacitaciones se recomienda utilizar la metodología denominada matriz de riesgo para realizar la evaluación de riesgo de las instalaciones radiactivas.

El método de matriz de riesgo surgió en el marco del Programa de Seguridad Radiológica, Nuclear y Física en Iberoamérica, de la Organización Internacional de Energía Atómica (OIEA) [3], en respuesta a la necesidad de prevenir las exposiciones potenciales en radioterapia. Este método plan- tea un análisis de riesgo sistemático y proactivo que permite determinar el perfil de riesgo de una instalación en cada una de las etapas de operación, considerando no solo eventos que hayan ocurrido en el pasado, sino también los que puedan ocurrir durante la operación normal de los equipos. La técnica de matriz de riesgo, en contraste con el método de modo y efecto de fallas (AMEF o FMEA, por sus siglas en inglés) [56], o la evaluación probabilística de seguridad [6-8], presenta una metodología de fácil uso e interpretación de resultados.

Debido al gran número de sucesos iniciadores (SI) asociados a las prácticas de radioterapia, por encima de 100 SI para braquiterapia HDR, se desarrolló la herramienta informática SEVRRA (System of Evaluation of Risk in Radiotherapy) [9]; con el objetivo de facilitar el análisis de riesgo utilizando la técnica de matriz de riesgo. La herramienta SEVRRA se ha aplicado a diferentes instalaciones de radioterapia, en especial, instalaciones de braquiterapia $\operatorname{HDR}[10,11]$. El análisis de riesgo mediante esta herramienta ha permitido obtener el perfil de riesgo de varias instalaciones de braquiterapia, y en función de la aceptabilidad de riesgo de la metodología, las instalaciones han podido priorizar las medidas que deben adoptarse con el objetivo de disminuir el riesgo a niveles aceptables.

En este trabajo se presenta el análisis de riesgo, con la metodología de matriz de riesgo, de una instalación de braquiterapia HDR. Dicho análisis se realizó utilizandola aplicación SEVRRA y una aplicación desarrollada en una hoja de cálculo. Ambas aplicaciones se basan en el método de matriz de riesgo [3]. Se presenta el perfil de riesgo obtenido con cada aplicación y se analizan las diferencias observadas. Adicionalmente, se discute la aceptabilidad del riesgo planteada en la metodología de matriz riesgo con base en los resultados obtenidos. 


\section{MATERIALES Y MÉTODOS}

El método de matriz de riesgo [4] es semicuantitativo, y con él se determina el perfil de riesgo de una práctica mediante el estudio del nivel de riesgo de una lista de situaciones potenciales que son activadas por eventos iniciadores asociados a cada etapa de operación de la práctica. La lista de eventos iniciadores para braquiterapia se ha realizado con base en publicaciones de accidentes o incidentes ocurridos en el pasado [12], e identificando qué puede fallar en cada una de las etapas de operación.

Cada situación potencial consiste en un evento iniciador que tiene una frecuencia de ocurrencia asociada $(f)$. El evento iniciador se produce por el fallo de las funciones de seguridad previstas en la práctica. Las funciones de seguridad pueden ser errores humanos o fallos de equipo que tienen una probabilidad de fallo asociada $(P)$. El fallo de las barreras conduce a que pacientes, trabajadores relacionados con los equipos, personal o público resulten expuestos a radiación. Dependiendo de la severidad de la exposición y del tipo de personal implicado el suceso iniciador tendrá un nivel de consecuencia asociado $(C)$.

El riesgo $(R)$ se determina combinando tres variables: frecuencia del evento iniciador $(f)$, probabilidad de fallo de las funciones de seguridad $(P)$ y el nivel de consecuencia $(C)$. Este último se determina planteando un escenario de exposición conservador donde todas las funciones de seguridad han fallado. En cada variable de la ecuación de riesgo $(R=f$ $\times \mathrm{P} \times \mathrm{C}$ ) se tienen cuatro niveles: muy alto (MA), alto $(\mathrm{A})$, medio (M) y bajo (B). La combinación de las tres variables se realiza mediante la denominada matriz de riesgo, que se presenta en la referencia [4]; de esta forma se determina el nivel de riesgo de cada evento iniciador.

El método plantea dos cribados. En el primero se tienen en cuenta el número de reductores de frecuencias y barreras; de esta forma, el nivel de riesgo de cada suceso iniciador es moderadamente conservador. Este primer análisis permite identificar las secuencias de mayor riesgo, lo que posibilita realizar un análisis detallado de esas secuencias. En el segundo cribado, aplicado a las secuencias con riesgo alto, se tiene en cuenta la robustez de las barreras y el número de reductores de frecuencia, lo que conduce bajo ciertas condiciones a realizar una reclasificación de la probabilidad de fallo $(P)$ y de la frecuencia del suceso iniciador $(f)$ y por lo tanto a reducir el nivel de riesgo de ciertas secuencias.
Teniendo en cuenta el resultado del perfil de riesgo obtenido luego de aplicado el segundo cribado, la metodología plantea criterios de aceptabilidad de riesgo y acciones correctoras para cada caso. Según la aceptabilidad de riesgo, si dentro del resultado se obtienen secuencias con riesgo muy alto (MA) se requiere paralizar la práctica y tomar medidas para reducir el riesgo antes de retomarla. En las secuencias con riesgo alto (A) se requieren tomar medidas inmediatas para reducir el riesgo cuando el suceso conlleva a consecuencias altas (A) o muy altas (MA). En las secuencias con consecuencias medias y bajas se requieren medidas a mediano plazo. En los demás casos, el nivel de riesgo es tolerable (riesgo medio) o despreciable (riesgo bajo), y las medidas que se tomen dependen del criterio costo/beneficio.

\subsection{Aplicación del método de matriz de riesgo a una instalación de braquiterapia HDR}

En base a la metodología de matriz de riesgo se realizó el análisis de riesgo con el software SEVRRA y con una aplicación desarrollada en una hoja de cálculo. En ambos casos se analizaron los mismos SI y se contó con las mismas funciones de seguridad. Se debe destacar que se adicionaron nuevas funciones de seguridad propias de la instalación, tanto para la aplicación SEVRRA como para la aplicación desarrollada.

Para determinar el nivel de riesgo de cada suceso iniciador con el método desarrollado en la hoja de cálculo, se siguió una metodología para asignar el nivel de consecuencia. La asignación de este nivel se realiza con el planteamiento de un escenario conservador - así se puede realizar la estimación de dosis de cada suceso iniciador- y con base en una escala definida para trabajadores ocupacionalmente expuestos y personas del público se asigna el nivel de consecuencia. Esta metodología se describe en detalle en el trabajo [13].

La descripción de los recursos de la instalación de braquiterapia HDR estudiada en este trabajo (BHDR-I), se expone en la tabla 1. Se debe destacar que la carga de trabajo definida en la tabla 1 (1.560 pacientes por año y cuatro recambios de la fuente radiactiva) se tiene en cuenta para estimar el nivel de frecuencia de los sucesos iniciadores según como se indica en la técnica de matriz de riesgo [4]. El nombre de la instalación bajo estudio no se revela con el objetivo de reservar sus datos. 
Tabla 1. Descripción de los recursos de la instalación de braquiterapia de alta tasa de dosis, denominada en este documento como BHDR-I.

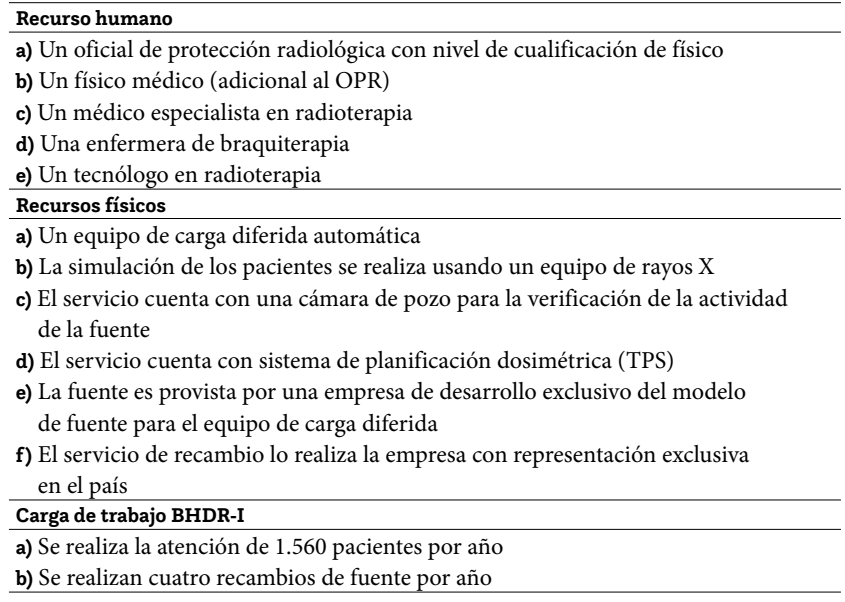

\section{RESULTADOS}

En la figura 1 se muestra el resultado del perfil de riesgo de la instalación de braquiterapia HDR BHDR-I, obtenido con la aplicación SEVRRA en contraste con el obtenido con la aplicación propia. Como se puede observar, el perfil de riesgo es considerablemente diferente, excepto para SI con riesgo muy alto, donde para las dos aplicaciones se obtiene un $0 \%$ de sucesos. Sin embargo, el porcentaje de sucesos con riesgo alto es de 6\% (5 sucesos) con la aplicación SEVRRA, y con la aplicación propia, 14\% (10 SI). El porcentaje de SI con riesgo medio es de $71 \%$ (56 SI) con SEVRRA, y 70\% (55 SI) con la aplicación propia. Finalmente, con riesgo bajo, para la aplicación SEVRRA se obtiene un 23\% (18 SI), en contraste con el 16\% (13 SI) para la aplicación propia.

Para las etapas de operación con sucesos con riesgo alto se observa la distribución de los sucesos en la figura 2, para la aplicación SEVRRA y la aplicación propia. En las etapas 1 y 2 (instalación inicial de los equipos y aceptación y puesta en servicio) no se obtienen sucesos de riesgo alto con la aplicación SEVRRA; en contraste, con la aplicación propia se encuentra 1 suceso con riesgo alto para la etapa 1 (Instalación inicial de los equipos) y 5 sucesos con riesgo alto para la etapa 2 (aceptación y puesta en servicio).

Para determinar la causa de las discrepancias encontradas se realizó un estudio detallado del nivel de riesgo en todos los sucesos iniciadores, y se encontró que las diferencias se deben a que la robustez asignada a las barreras en la aplicación SEVRRA no coincide con la definida en el método de matriz de riesgo. En particular, el nivel de riesgo alto obteni-

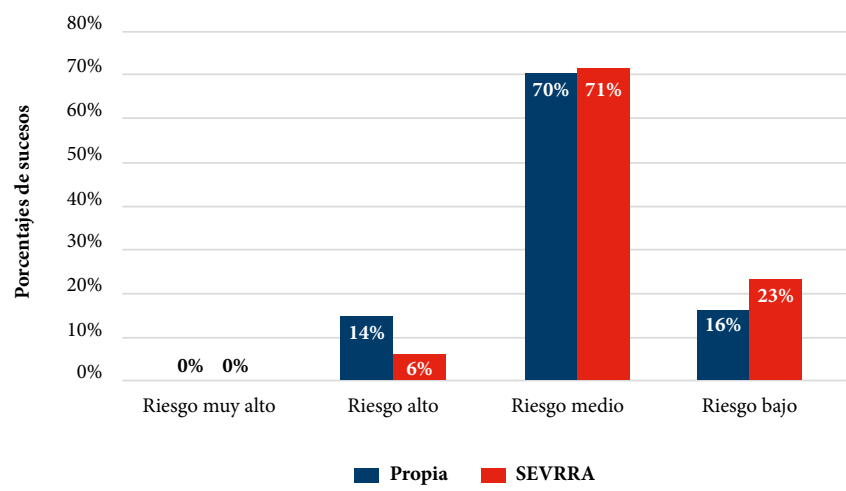

Figura 1. Comparación del perfil de riesgo de una instalación de braquiterapia BHDR-I, realizado con en base a la técnica de matriz de riesgo, utilizando la aplicación SEVRRA y una aplicación propia.

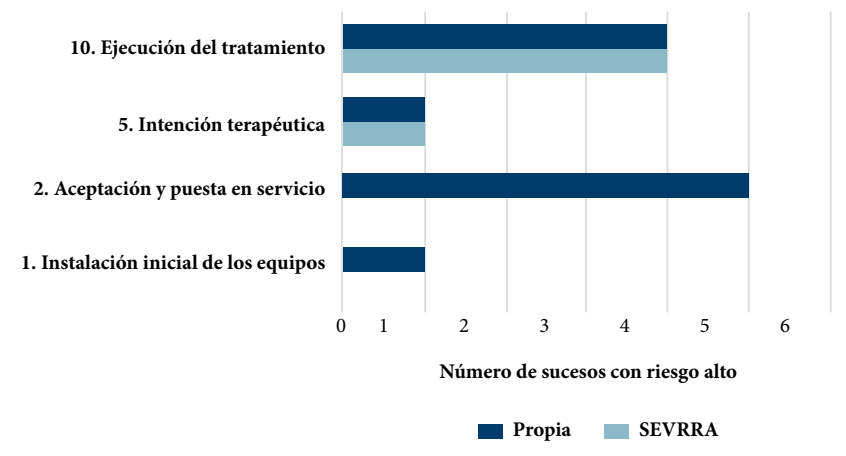

Figura 2. Distribución de sucesos iniciadores con riesgo alto por etapa de operación, en la instalación de braquiterapia BHDR-I. Se debe resaltar que solo aparecen las etapas de operación para las que se obtienen secuencias con riesgo alto.

do en los seis sucesos, en las etapas 1 y 2 (instalación inicial de los equipos y aceptación y puesta en servicio) se debe a que para la barrera "Calibración de la fuente durante la puesta en servicio y comparación del resultado con el valor de tasa de kerma que consta en el certificado", en la aplicación propia se asignó una robustez de 4 , mientras en la aplicación SEVRRA se asignó una robustez de 32. Esto difiere a lo estipulado en el método de matriz de riesgo, donde en los apéndices [14] se asigna a esta barrera una robustez de 4, valor igual al asignado para esta barrera en la aplicación propia.

De esta forma, la diferencia del perfil de riesgo en las dos aplicaciones se debe a la diferencia de robustez asignada a algunas barreras, como se evidencia en la tabla 2. Adicionalmente, en dicha tabla se muestra la robustez asignada en los apéndices del método de matriz de riesgo [14]. Como se puede observar, se encuentran diferencias entre la robustez asignada en cada método a ciertas barreras; esto se debe a la interpretación equivocada del tipo de barreras, lo que ha sido reportado en anteriores trabajos [11]. 
Tabla 2. Barreras en las que la aplicación SEVRRA tiene una robustez diferente a la asociada en la metodología de matriz de riesgo del TECDOC-1685/S [13]

\begin{tabular}{lcc}
\hline \multicolumn{1}{c}{ Descripción de la barrera } & Robustez en SEVRRA & Robustez en apéndices TECDOC-1685/S \\
\hline $\begin{array}{l}\text { Calibración de la fuente durante la puesta en } \\
\text { servicio y comparación del resultado con el valor de } \\
\text { tasa de kerma que consta en el certificado }\end{array}$ & 32 & 4 \\
\hline $\begin{array}{l}\text { Confrontación de los datos importados por el panel } \\
\text { de control con los reportados por el TPS, que han } \\
\text { sido registrados en el plan de tratamiento }\end{array}$ & 32 & 4 \\
\hline $\begin{array}{l}\text { Experiencia del operador relativa a los tiempos } \\
\text { ordinarios de tratamiento }\end{array}$ & 8 & No reporta \\
\hline $\begin{array}{l}\text { Monitoreo redundante del paciente utilizando un } \\
\text { detector portátil de radiación }\end{array}$ & 8 & 4 \\
\hline
\end{tabular}

La interpretación equivocada podría atribuirse a que el usuario puede asociar a barreras tipo 2 (alarmas) una robustez de una barrera tipo 4 (procedimientos), cuando la alarma hace parte de un procedimiento realizado por una persona, lo que no corresponde con la definición en el método de matriz de riesgo.

El hecho de asociar diferente robustez tiene un gran impacto, ya que puede causar un efecto considerable en el perfil de riesgo de la instalación, como se evidencia en este trabajo. Esto sugiere que en la metodología de matriz de riesgo se debería realizar una definición más amplia de cada tipo de barrera, con el fin de que las instalaciones puedan asignar el nivel adecuado de robustez.

\section{ACEPTABILIDAD DE RIESGO}

Para realizar el análisis de la aceptabilidad del riesgo se tomaron los resultados del perfil de riesgo obtenidos con la aplicación propia. En la tabla 3 se encuentran descritos los sucesos iniciadores con nivel de riesgo alto, así como el nivel de consecuencia para dichos sucesos. Como se puede observar, todos estos sucesos tienen consecuencias altas o muy altas, por lo que, según la aceptabilidad del riesgo de la metodología, se deberían tomar medidas inmediatas para reducir el riesgo. Por ello, en la mencionada tabla se presentan las medidas de seguridad que se deberían tomar en la instalación de braquiterapia para reducir el riesgo. Para los sucesos asociados a las etapas 1 y 2 (instalación inicial de los equipos y aceptación y puesta en servicio) así como en la etapa 5 (intención terapéutica), para reducir el riesgo se deben introducir barreras de verificación redundante, que se describen a continuación:

Verificación de los resultados de la calibración, de manera independiente redundante, por otro físico médico y con otro sistema dosimétrico.

Verificación de los cálculos resultantes de la planificación dosimétrica del tratamiento mediante cálculo inde- pendiente realizado por un físico-médico diferente del que planificó el caso.

Por otra parte, para reducir el riesgo del SI BHDR-PAC9.11 se deben introducir, como reductor de frecuencia, el registro en las planillas para la recogida y reporte de información del tratamiento.

Estas funciones de seguridad permitirían reducir el nivel de riesgo de nivel alto a medio en estos sucesos, con lo que se lograría ajustar la instalación al criterio de aceptabilidad de riesgo del método de matriz de riesgo.

Como se ha evidenciado, se deben introducir barreras de seguridad de tipo verificación redundante; sin embargo, se debe destacar que la aplicación de estas funciones de seguridad en instalaciones pequeñas ubicadas en zonas poco pobladas del país, y con limitado personal, no es viable en la mayoría de los casos, si se considera el criterio de costo/ beneficio. Esto implicaría que estas instalaciones no podrían seguir operando. Por esta razón se debe buscar otro tipo de barreras, con el fin de que sea viable la operación segura de esta clase de instalaciones y de garantizar su operación en el nivel de tolerabilidad del riesgo.

Es importante destacar que la metodología de matriz de riesgo no contempla el riesgo inherente a cada práctica en el marco de la aceptabilidad de riesgo. Esto puede tener consecuencias negativas cuando los reguladores de los países se basen en la aceptabilidad de riesgo para emitir o negar autorizaciones de manejo de material radiactivo. Como evidencia en este trabajo, para tres SI (BHDR-PAC9.6, BHDR-PAC9.13 y BHDR-PAC9.15) no se puede mitigar el riesgo, ya que no es posible reducir la frecuencia ni la probabilidad de fallo. Específicamente, en estos tres sucesos no se conocen barreras, tal como se pone en evidencia en los apéndices del método de matriz de riesgo [14]. El riesgo inherente a la práctica indicaría $4 \%$ de sucesos iniciadores con nivel de riesgo alto (3 SI de un total de 79 SI analizados). 
Tabla 3. Secuencias con riesgo alto obtenidas con la aplicación propia

\begin{tabular}{|c|c|c|}
\hline Descripción del suceso & Nivel de consecuencia & Medidas que se deben adoptar \\
\hline BHDR-PAC1.3. Error en el certificado del fabricante de la fuente & MA & Verificación redundante \\
\hline $\begin{array}{l}\text { BHDR-PAC2.1. Error de calibración de la cámara y el electrómetro (en el } \\
\text { laboratorio de patrones de dosimetría) }\end{array}$ & MA & Verificación redundante \\
\hline $\begin{array}{l}\text { BHDR-PAC2.2. Error de calibración de la cámara y el electrómetro (en el } \\
\text { laboratorio de patrones de dosimetría) }\end{array}$ & MA & Verificación redundante \\
\hline $\begin{array}{l}\text { BHDR-PAC2.4. Error de interpretación de las cifras decimales del } \\
\text { temporizador del equipo de braquiterapia y/o electrómetro durante la } \\
\text { calibración de la fuente }\end{array}$ & MA & Verificación redundante \\
\hline BHDR-PAC2.5. Utilización de valores incorrectos de decaimiento de la fuente & MA & Verificación redundante \\
\hline $\begin{array}{l}\text { BHDR-PAC2.7. Error en la determinación de la tasa de kerma en aire en } \\
\text { condiciones de referencia }\end{array}$ & MA & Verificación redundante \\
\hline $\begin{array}{l}\text { BHDR-PAC4.3. Registrar por error un valor de dosis total de tratamiento, } \\
\text { dosis por fracción o fraccionamiento diferente del valor prescrito en la hoja de } \\
\text { tratamiento }\end{array}$ & A & Verificación redundante \\
\hline $\begin{array}{l}{ }^{*} \text { BHDR-PAC9.6. Desconexión de la fuente del cable de transferencia al efectuar } \\
\text { un tratamiento }\end{array}$ & A & Reducir las consecuencias \\
\hline $\begin{array}{l}\text { BHDR-PAC9.11. Error al registrar los datos de la sesión diaria en la hoja de } \\
\text { tratamiento }\end{array}$ & A & Introducir reductor de frecuencia \\
\hline $\begin{array}{l}\text { *BHDR-PAC9.13. Desconexión de la fuente del cable de transferencia, } \\
\text { permaneciendo en un implante intracavitario o superficial, tras finalizar el } \\
\text { tratamiento }\end{array}$ & A & Reducir las consecuencias \\
\hline $\begin{array}{l}{ }^{\star B H D R}-\mathrm{PAC} 9.15 \text {. Atascamiento de la fuente tras finalizar el tratamiento, } \\
\text { permaneciendo fuera del paciente y del blindaje }\end{array}$ & A & Reducir las consecuencias \\
\hline
\end{tabular}

${ }^{\star}$ Sucesos para los que no se puede mitigar el riesgo.

\section{CONCLUSIONES}

La aplicación del método de matriz de riesgo permite la evaluación del perfil de riesgo en la práctica de braquiterapia HDR. Debido al número elevado de sucesos iniciadores en la práctica (> 70), es necesario usar aplicaciones que faciliten la evaluación del perfil de riesgo de las instalaciones. El software SEVRRA, aún en desarrollo, facilita considerablemente esta labor. Presenta una interfaz amigable que permite evaluar los resultados y estudiar la eficacia de las funciones de seguridad. Sin embargo, deben tomarse precauciones en su aplicación, ya que en la versión actual algunas barreras no tienen la robustez considerada en la metodología de matriz de riesgo.

Como se expuso en este trabajo, los resultados del perfil de riesgo con la aplicación SEVRRA no coincidieron con los obtenidos con la herramienta propia, a pesar de que las dos aplicaciones están basadas en la misma metodología. La diferencia encontrada, se debe a que la robustez asignada para algunas barreras por el software SEVRRA es más alta que la definida por la técnica de matriz de riesgo. Este problema puede ser fácilmente solucionado por los diseñadores de la aplicación SEVRRA cambiando la robustez de las barreras, o por los usuarios, creando nuevas barreras con la misma descripción, pero asignando la robustez correspondiente de tal forma que coincida con la definida por la metodología de matriz de riesgo.
Otra precaución que se debe tener en cuenta para utilizar el método de matriz de riesgo se centra en la interpretación de los resultados, debido a que la metodología plantea una aceptabilidad de riesgo conservadora. Sin embargo, esta consideración no tiene en cuenta los riesgos inherentes a la práctica, esto es, sucesos iniciadores que hasta el momento no tengan barreras conocidas y que podrían tener riesgo alto. El ente regulador o evaluador debe tener en cuenta este hecho a la hora de analizar el perfil de riesgo de una instalación.

Se debe destacar que el método de matriz de riesgo ha permitido la determinación del perfil de riesgo de instalaciones de braquiterapia en el país, gracias a esto las instalaciones han fortalecido sus sistemas de calidad y de seguridad física. Adicionalmente, las instalaciones han reconocido la importancia de las capacitaciones del personal, tanto en protección radiológica como en actividades asociadas a la operación normal de la práctica, así como en la respuesta a situaciones accidentales que surgen en la práctica.

\section{Referencias}

[1] S. C. B. Esteves, A. C. Z. de Oliveira, and L. F. de A. Feijó, "Braquiterapia de alta taxa de dose no Brasil," Radiologia Brasileira, vol. 37, no. 5, pp. 337-341, 2004. https://doi. org/10.1590/S0100-39842004000500007 
[2] IAEA, Radiation protection and safety of radiation sources: International basic safety standards. IAEA Safety Standards Series No. GSR, Part 3, Viena: IAEA, 2014.

[3] Ministerio de Minas y Energía (Colombia), Resolución 90874 de 2014. https://www.minenergia.gov.co/documents/10192/0/90874.pdf/ba4e5ee5-dc42-41d0-85931f83fa59f3b4

[4] IAEA, Aplicación del método de matriz de riesgo a la radioterapia. Viena: IAEA-TECDOC 1685/S, Organismo Internacional de Energía Atómica, 2010. Disponible en: https://www-pub.iaea.org/MTCD/Publications/PDF/ TE_1685_S_CD/PDF/Appendix.pdf

[5] D. H. Stamatis, Failure modes and effects analysis. Milwaukee, WI: American Society for Quality Control, 1995.

[6] P. Ortiz López, "Tools for risk assessment in radiation therapy," Annals of the ICRP, vol. 41, no. 3-4, pp. 197-207, Oct. 2012. https://doi.org/10.1016/j.icrp.2012.06.025

[7] IAEA, Case studies in the application of probabilistic safety assessment: Techniques to radiation sources. IAEA TECDOC, no. 1494. Viena: International Atomic Energy Agency, 2006.

[8] IAEA, Análisis probabilista de seguridad de tratamientos de radioterapia con acelerador lineal. IAEA TECDOC, no. 1670/S. Viena: International Atomic Energy Agency, 2012.
[9] Foro Iberoamericano de Organismos Reguladores y Nucleares, "SEVRRA". [Online]. Disponible en https:// www.foroiberam.org/sevrra. (Consultado en 2018).

[10]D. J. McDonnell, S. B. Papadópulos, A. B. Paz, R. López Morones y F. Ramírez Pérez, "Aplicación de SEVRRA para la evaluación de condiciones de riesgo en braquiterapia HDR", Presentado en el IX Congreso Regional de Seguridad Radiológica y Nuclear. Río de Janeiro, Brasil, 15 al 19 de abril, 2013.

[11]C. Duménigo et al., "Application of the risk matrix approach in radiotherapy: An Ibero-American experience". Presentado en el Foro Iberoamericano de Organismos Reguladores Radiológicos y Nucleares, 2014.

[12] IAEA, Lessons learned from accidental exposures in radiotherapy. SRS n. ${ }^{\circ}$ 17. Viena: Organismo Internacional de Energía Atómica, 2002.

[13] M. Arciniegas Álvarez, D. C. Herrera, J. A. Gómez y A. Estrada Molina, "Análisis de riesgo de instalaciones de radioterapia y medicina nuclear en Colombia con el método de matriz de riesgo". Presentado en el XI Congreso Regional de Seguridad Radiológica y Nuclear, La Habana, Cuba, 16-20 de abril, 2018.

[14] IAEA, Aplicación del método de matriz de riesgo a la radioterapia. IAEA-TECDOC 1685/S: Apéndices. Viena: Organismo Internacional de Energía Atómica, 2012. 